\title{
Modelling Approach and Challenges in Simulating Dross Formation in Ductile Iron Castings
}

\author{
Seyyed Hojjat Majidi ${ }^{1, a}$, Christoph Beckermann ${ }^{1, b}$, Jakob Fainberg ${ }^{2, c}$, \\ Wilfried Schäfer ${ }^{2, d}$, Mathias Bodenburg ${ }^{2, e^{*}}$ \\ ${ }^{1}$ Department of Mechanical and Industrial Engineering, University of lowa, lowa City, \\ IA 52242, USA \\ 2MAGMA Gießereitechnologie GmbH, Kackertstrasse 11, 52072 Aachen, Germany \\ ahojjat-majidi@uiowa.edu, bbecker@icaen.uiowa.edu, cj.fainberg@magmasoft.de, \\ dw.schaefer@magmasoft.de, ${ }^{e}$ m.bodenburg@magmasoft.de
}

Keywords: Dross Formation, Air Entrainment, Ductile Iron, Simulation of Casting Process

\begin{abstract}
Dross is one of the most challenging quality issues in high integrity ductile iron castings. The formation of dross is linked to the applied metallurgy, metal treatment, process control and gating/rigging design. One of the primary concerns in this regard is entrainment of air into the melt during filling of the casting. Today, avoiding and controlling dross relies on the practical experience and process know-how of foundry experts. A quantitative understanding of the formation mechanisms or the prediction of final amounts and locations of dross in castings is not available.

In this paper a computational model is developed for predicting the formation, motion and final location of dross inclusions during pouring of ductile iron castings. The focus is on the important mechanism of dross generation due to air entrainment during filling of the mold. The model predicts the local air entrainment rate as a function of the turbulent kinetic energy of the liquid metal and the normal velocity gradient of the metal at the liquid metal-air interface. The dross inclusions resulting from the exposure to the entrained air are transported with the melt flow under the combined influences of drag and buoyancy, and captured by the solidifying casting structure. The model is implemented in a commercial casting simulation software code.
\end{abstract}

\section{Introduction}

Dross inclusions are commonly reported in ductile iron castings. They form after magnesium treatment during holding and melt transfer, and further during pouring of liquid metal. Air entrainment during filling is the main source of the oxygen that is consumed in dross formation. The quantitative comparison of measured and simulated dross is a challenge as usually only the area where dross occurs is indicated on a real part, and normally this affected area doesn't completely consist of dross inclusions (Figure 1). Usually ultrasonic inspection locates the affected areas but does not give a good indication of the amount of dross. Therefore a quantitative comparison requires a clear guideline how to assess those areas.

In this paper a computational model is developed for predicting the formation, motion and final location of dross inclusions in ductile iron castings. The developed model predicts the local air entrainment at free surface discontinuities. Based on the predicted entrained air volume, inclusions are generated at the liquid metal-air interface, transported with the melt flow under the combined influences of drag and buoyancy, and captured by the solidifying casting surface. The model is implemented in a commercial casting simulation software code. Ductile iron casting experiments have been conducted to validate the model. 


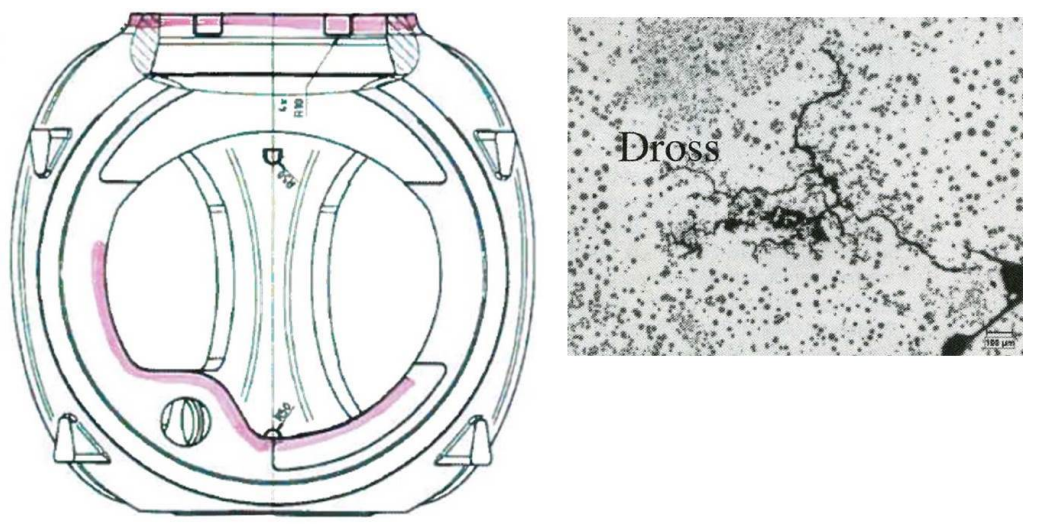

Figure 1: Typical marks to indicate areas of dross and corresponding microstructure.

\section{Modelling Approach of Dross Formation in Ductile Iron Castings}

Dross consists mainly of silica, $\mathrm{SiO}_{2}$, and magnesia, $\mathrm{MgO}$, and the combination of these two oxides, i.e. magnesium silicates in the form of $2 \mathrm{MgO}^{*} \mathrm{SiO}_{2}$ (forsterite). In addition, $\mathrm{Al}_{2} \mathrm{O}_{3}, \mathrm{CaO}$ and traces of sulfides can also be found in the composition of dross inclusions.

When treating an iron melt with magnesium to achieve a nodular structure of the graphite during solidification, the formation of these oxides and the resulting dross formation cannot be avoided. Magnesium has a high affinity for oxygen; hence, as soon as the nodularizer reacts with the base iron, the reaction between the magnesium and the oxygen inside the melt and the surrounding air forms $\mathrm{MgO}$. On the other hand, $\mathrm{SiO}_{2}$ forms during cooling of the melt and the carbo-thermic reduction of silica seems to play an important role in the formation of silica:

$$
<\mathrm{SiO}_{2}>+2[\mathrm{C}] \rightarrow[\mathrm{Si}]+2\{\mathrm{CO}\}
$$

Figure 2 shows the isotherms of silica reduction by carbon for different temperatures. At melt temperatures above the equilibrium temperature, carbon dominates the oxygen content of the melt, and silica $\left(\mathrm{SiO}_{2}\right)$ deoxidizes forming carbon monoxide gas. However, at temperatures below the equilibrium temperature silicon burns off and $\mathrm{SiO}_{2}$ forms. The higher the equilibrium temperature, the sooner the melt reaches this temperature during cooling and the higher the risk of silicon oxide formation, and consequently formation of dross inclusions.

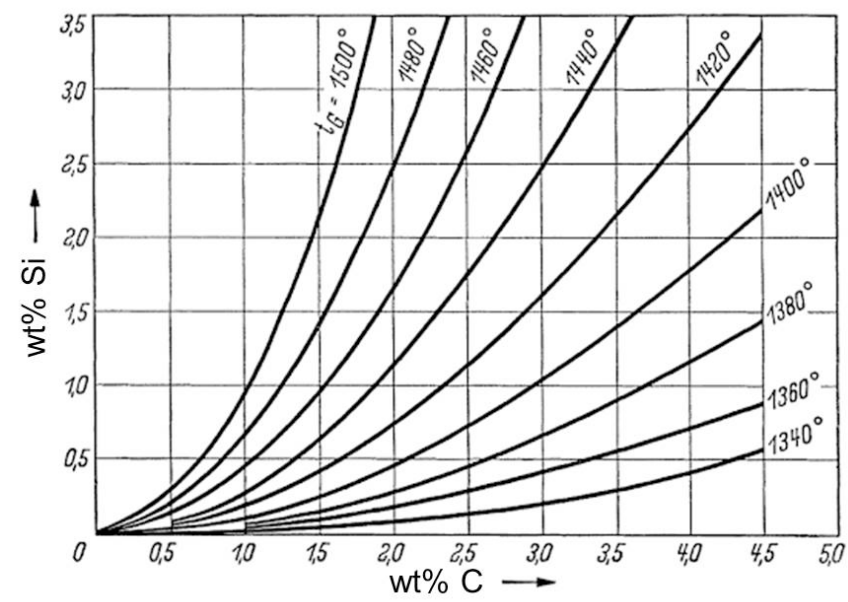

Figure 2: Equilibrium isotherms of silica reduction by carbon [1].

This probably explains why dross is mainly a problem in large castings. Large parts are usually cast with a relatively low pouring temperature to reduce the size and number of feeders or even get a sound casting without any risers. In addition, foundrymen aim to lower the thermal impact to the molding material for avoiding metal-mold reactions and the resulting defects. Moreover, due to the longer filling times in large parts, longer times are allowed for the potential oxidation of silicon. 
Besides to the metallurgical effects (chemical composition, Mg-treatment, inoculation) and the melting practice (furnace, refractory, thermal history), a very important aspect is the quality of the filling process, and the contact time of the melt with air. This has been the principal motivation to develop an air entrainment model for predicting dross formation during filling. In this model the local air entrainment rate is calculated as a function of the turbulent kinetic energy and the magnitude of the normal velocity gradient of the liquid metal at the liquid-air interface [2].

\section{Air Entrainment Model}

The present model is based on the recent work of Ma et al. [3] in which the local air entrainment rate is calculated as a function of the turbulent kinetic energy and the magnitude of the normal velocity gradient at the liquid-air interface. The turbulence inside the liquid jet results in rough liquid-gas interface. These surface roughnesses contain air pockets with an average size of $\delta$, Figure 3. Air is entrained in a layer close to the interface where these surface roughnesses exist. The volumetric air entrainment rate per unit interfacial area is estimated by the amount of air passing the inward surface, $\Gamma_{\mathrm{e}}$, relative to the liquid-gas interface, $\Gamma_{\mathrm{i}}$ :

$$
q(\boldsymbol{x})=\frac{C_{\mathrm{ent}}}{g} k(\boldsymbol{x}) \frac{\partial u_{n}}{\partial n}(\boldsymbol{x})
$$

where $C_{\text {ent }}$ is a dimensionless entrainment coefficient that has to be determined, and $k\left(\mathrm{~m}^{2} / \mathrm{s}^{2}\right)$ and $g$ $\left(\mathrm{m} / \mathrm{s}^{2}\right)$ are the turbulent kinetic energy and the gravitational acceleration, respectively [3]. $\partial u_{n} / \partial n$ is the normal derivative of the normal component of the liquid velocity at the interface. In this model only positive values of $\partial u_{n} / \partial n$ will lead to air entrainment.

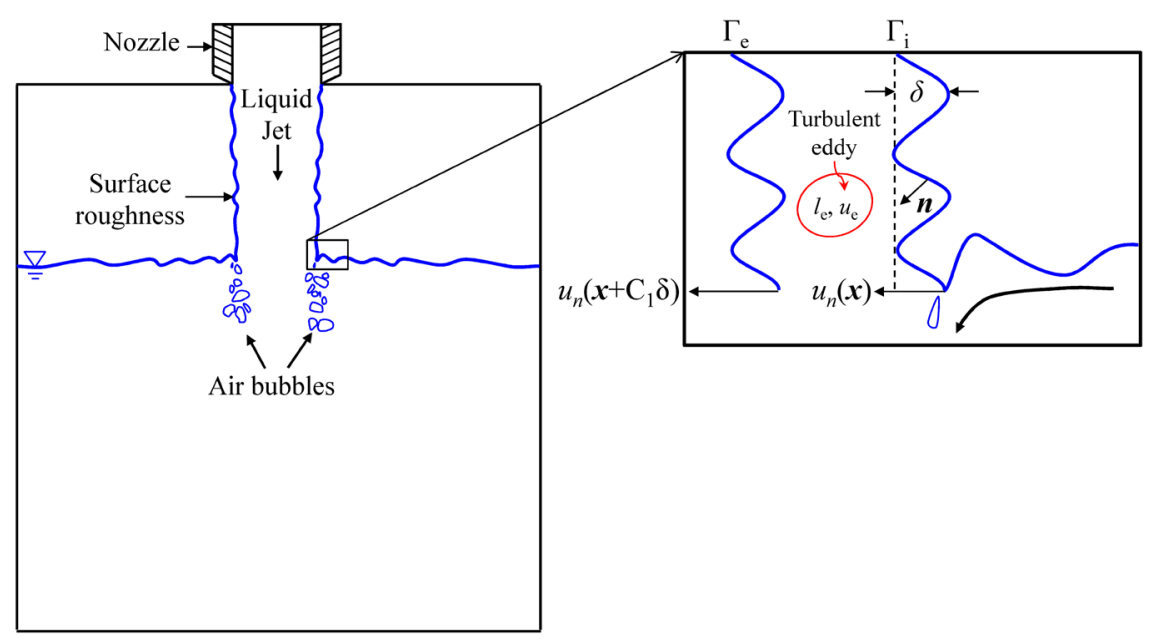

Figure 3: Schematic of air entrainment at liquid-gas interface, adapted from [2].

Integrating the rate of air entrainment per unit interfacial area over the interfacial area gives the volumetric air entrainment rate, $Q_{a}\left(\mathrm{~m}^{3} / \mathrm{s}\right)$ :

$$
Q_{a}=\iint_{A_{s}} q \mathrm{~d} A
$$

where $A_{s}$ is the interfacial area.

Experimental data [4] and [5] were used to validate the air entrainment model and to determine the entrainment coefficient, $C_{\text {ent }}$ by comparing the predicted steady state relative air entrainment rates to experimental measurements for different liquid jet velocities and diameters: $C_{\text {ent }}=0.039$. Details of the calibration can be found in the paper published by Majidi and Beckermann [2]. 


\section{Dross Formation, Agglomeration and Tracking Model}

The present model assumes that the oxygen available in the entrained air immediately reacts with the melt constituents (iron plus alloying elements). For estimating the quantity of dross inclusions, first the volume of entrained air must be calculated. This can be achieved by integrating the volumetric air entrainment rate, $Q_{a}$, over time for each computational domain:

$$
V_{a}=\int_{t_{0}}^{t_{0}+\Delta t} Q_{a}(t) \mathrm{d} t
$$

where $V_{a}, Q_{a}$ and $\Delta t$ are the volume of entrained air, volumetric air entrainment rate and the time interval between two inclusion generations, respectively.

A conversion constant, $C_{\mathrm{cnv}}$, is required for converting the volume of entrained air to the volume of inclusion. This constant is a function of the chemical composition of the inclusion. A typical composition of dross inclusion is shown in table 1 . The molecular weight is $96.9 \mathrm{~g} / \mathrm{mol}$ and the density is calculated as $3.01 \mathrm{~g} / \mathrm{cm}^{3}$ at $1400{ }^{\circ} \mathrm{C}$.

Table 1: Molecular weights and mole fractions of a characteristic dross composition

\begin{tabular}{lcc} 
Element & $\begin{array}{c}\text { Molecular Weight } \\
(\mathrm{g} / \mathrm{mol})\end{array}$ & Mole Fraction \\
\hline $\mathrm{MgSiO}_{3}$ & 100.4 & 0.369 \\
$\mathrm{Mg}_{2} \mathrm{SiO}_{4}$ & 140.7 & 0.22 \\
$\mathrm{FeO}$ & 71.8 & 0.199 \\
$\mathrm{Al}_{2} \mathrm{O}_{3}$ & 102.0 & 0.056 \\
$\mathrm{CaO}$ & 56.1 & 0.142 \\
$\mathrm{MnO}$ & 70.9 & 0.015
\end{tabular}

Assuming air at atmospheric pressure, $P=1013$ mbar, and pouring temperature of $T=1400{ }^{\circ} \mathrm{C}$, the inclusion volume, $V_{\mathrm{inc}}$, and diameter, $d_{\text {inc }}$, are calculated as:

$$
\begin{aligned}
& V_{\text {inc }}=\frac{m_{\text {inc }}}{\rho_{\text {inc }}}=\frac{n_{\text {inc }} M_{\text {inc }}}{\rho_{\text {inc }}} \quad ; V_{\text {air }}=\frac{n_{\mathrm{O}_{2}} \overline{\mathrm{R}} T}{0.2095 \times P} \\
& C_{\text {cnv }}=\frac{V_{\text {inc }}}{V_{\text {air }}}=4.9 \times 10^{-5} \frac{n_{\text {inc }}}{n_{\mathrm{O}_{2}}}=39.1 \times 10^{-6} \\
& V_{\text {inc }}=C_{\text {cnv }} \times V_{\text {inc }} ; \quad d_{\text {inc }}=\left(\frac{6}{\pi} V_{\text {inc }}\right)^{\frac{1}{3}}
\end{aligned}
$$

where $m_{\text {inc }}, \rho_{\text {inc }}$ and $n_{\text {inc }}$ are the mass, density and the number of moles of inclusion, respectively. Inclusions are generated at the mass center of the entrained domain and are initialized with the local flow velocity. They can agglomerate only at generation times. Figure 4 shows how agglomeration of inclusions is considered. Assuming that each inclusion corresponds to a bubble in the flow domain, a reasonable agglomeration length, $L_{\mathrm{agg}}$, of $2 \mathrm{~mm}$ was determined by comparing simulations with the bubble size distribution of a set of experiments [6]. 


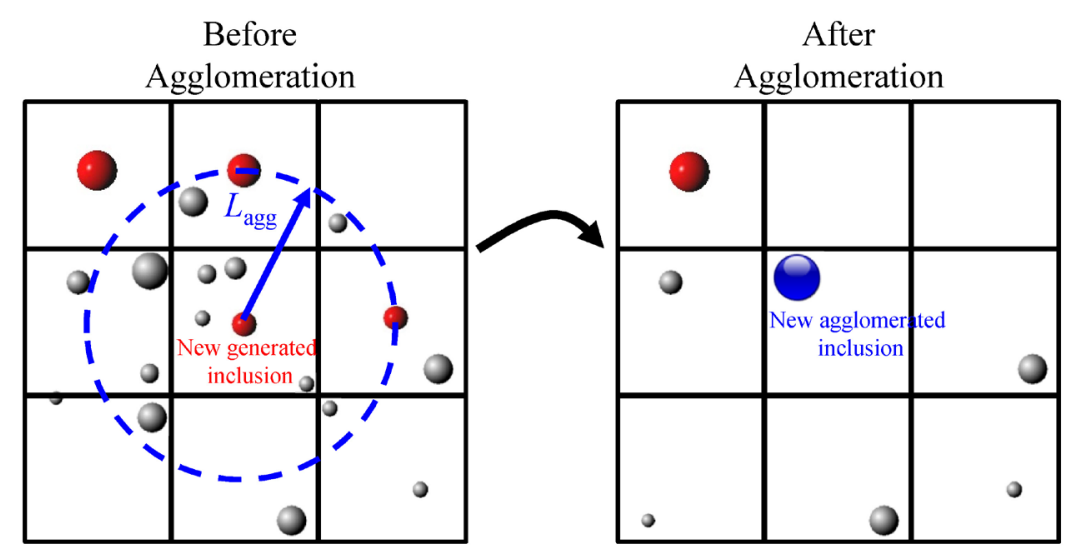

Figure 4: Agglomeration of Inclusions.

The final location of inclusions is determined by solving the equation of motion for each inclusion at each time step. Drag and buoyancy are the only forces acting on the inclusions.

\section{Validation of the Model}

Figure 5 shows the geometries of a test casting with different gating systems. Air is entrained during filling, the oxygen inside the entrained air reacts with the liquid metal generating dross inclusions. These inclusions grow and are carried away by the liquid metal flow. Figure 6 shows the final location of dross inclusions (a) and the respective area fraction of dross inclusions close to the surface of the casting (c). For detecting the inclusions layer by layer, the test castings were examined carefully by milling the surface in $1 \mathrm{~mm}$ steps down to a depth of $8 \mathrm{~mm}$ (b). Comparison between simulated and measured (indications in all layers) surface defects reveals a qualitatively good agreement.

\section{Discussion of the New Model}

In the described model, dross formation solely depends on the local air entrainment. The oxygen available in the entrained air then reacts with the liquid metal constituents to form dross inclusions. Comparisons with real castings indicate that the amount of dross generated due to air entrainment is sometimes less than the dross inclusions found in production castings. One reason could be that dross is already present in the melt before pouring. In addition, as mentioned earlier dross continuously forms during holding time as the melt temperature gets below the equilibrium temperature of the silica reduction by carbon (Figure 2). Another aspect, especially in large castings, is the natural convection which plays an important role in determining the final location and distribution of dross inclusions. Therefore it is important to take the convective movement during solidification into account to match the real situation.

Figure 7 shows the filling of a ladle. The inclusions are mainly generated where the metal stream impinges at the surface of the melt. The final distribution of the inclusions at the end of filling the ladle and after an additional 10 min holding time are shown in figure 8. Due to large buoyancy and convective currents, dross inclusions often accumulate at the top surface of the melt. In practice most of the formed oxides are considered as slag and are removed frequently from the melt surface prior to pouring.

The simulation results also reveal that smaller inclusions remain inside the melt as they are small enough to be carried by the flow. Moreover, dross continuously forms as the temperature of the melt gets below the above mentioned equilibrium temperature (Figure 2). Therefore, it is likely that even the deslagged melt carries a substantial number of small dross particles. If the ladle isn't 

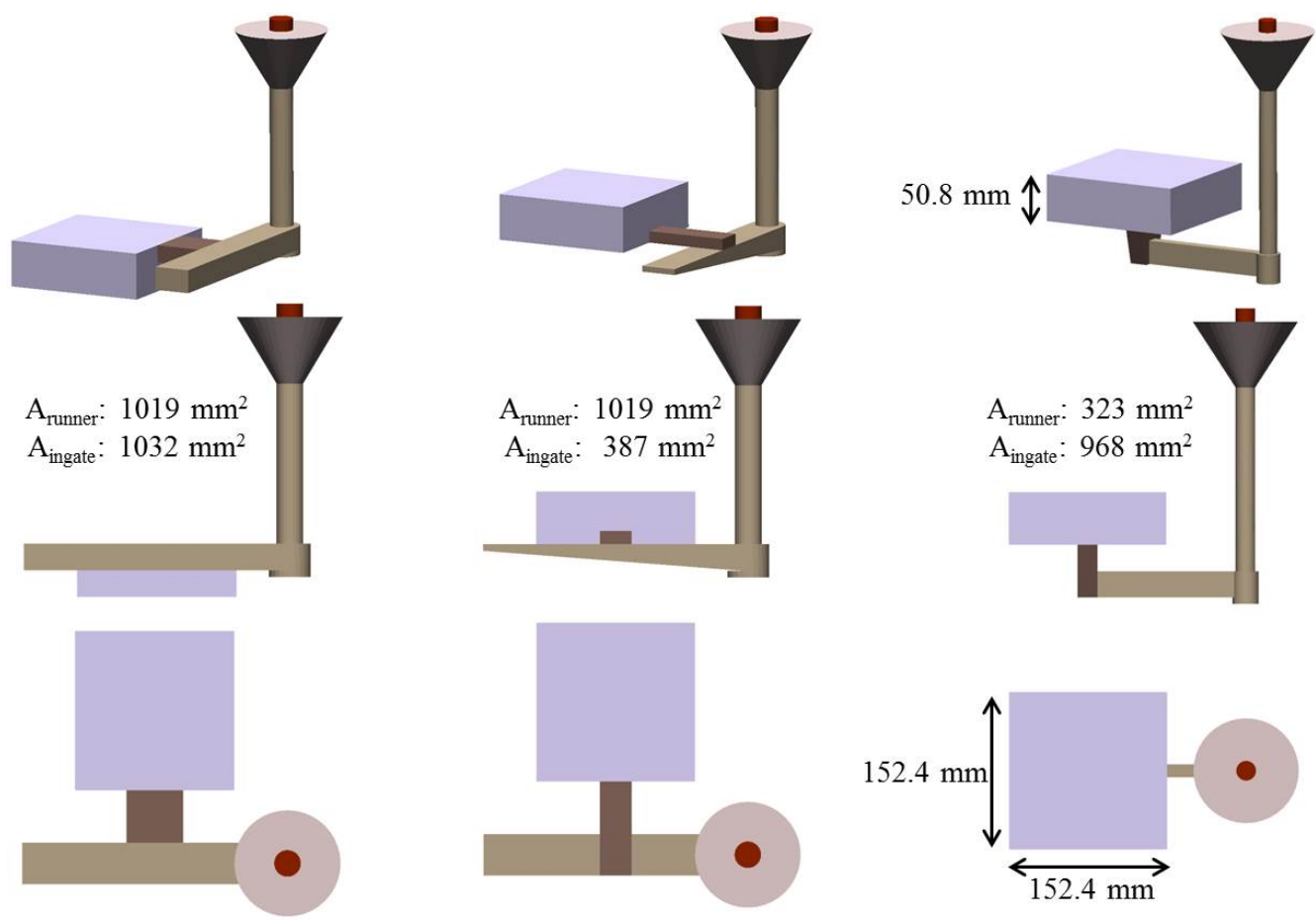

Figure 5: Test casting with three different gating systems.

a)
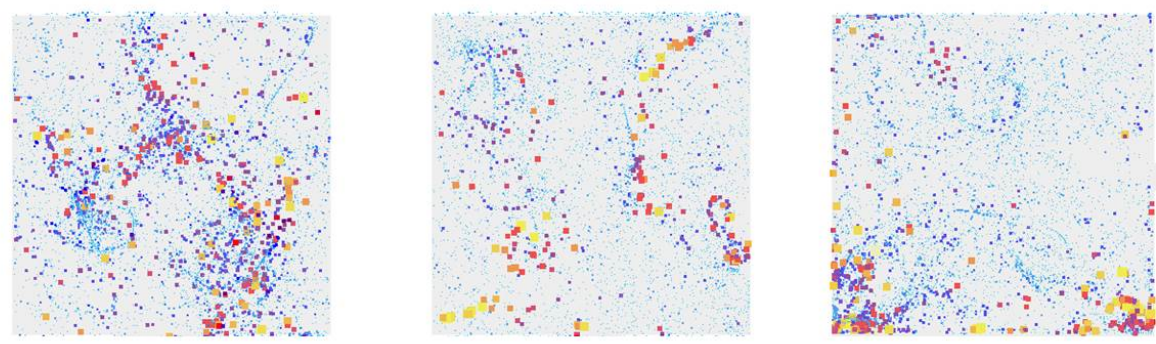

b)
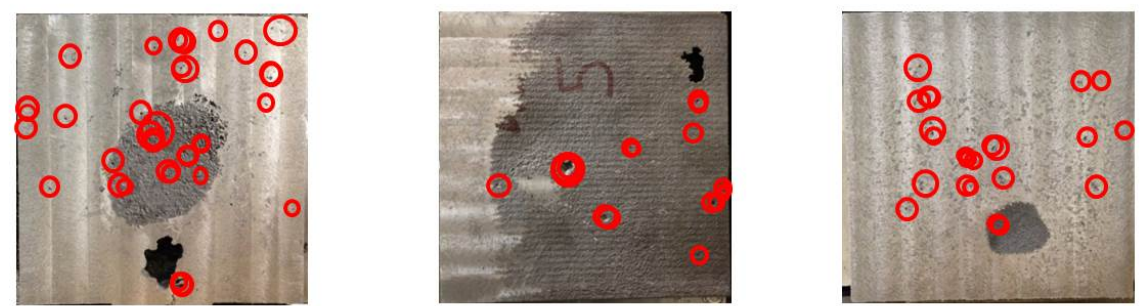

c)
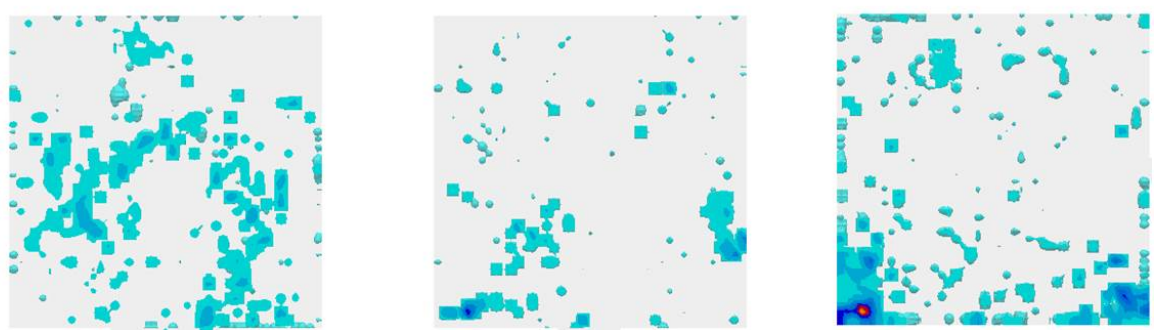

Figure 6: Comparison of measured (b) and simulated dross inclusions (a, c).

tapped carefully the inclusions will enter the mold cavity during pouring of the casting. In this case even argon or nitrogen atmospheres in the mold won't be able to avoid dross formation completely. This creates a challenge to characterize the melt in terms of dross formation (dross 

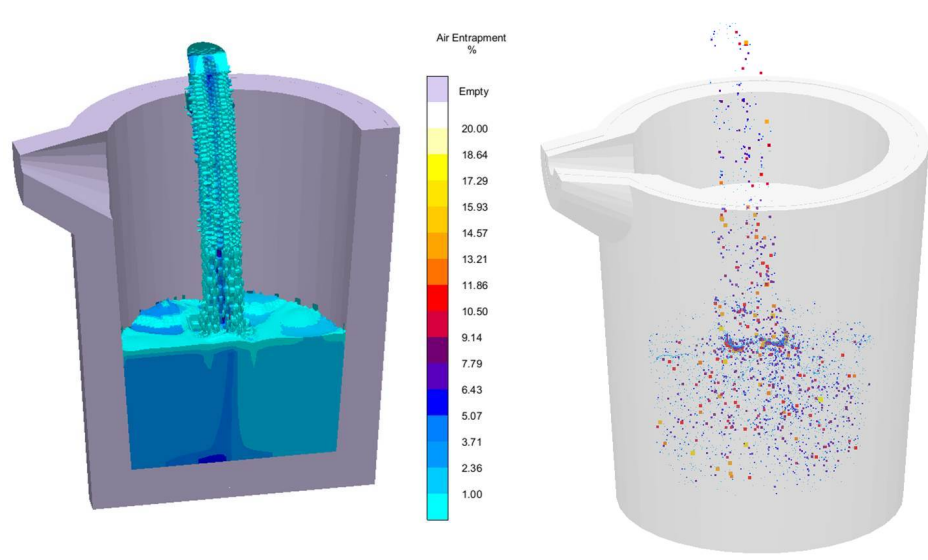

Figure 7: Air entrainment and formation of inclusions during filling of a ladle.
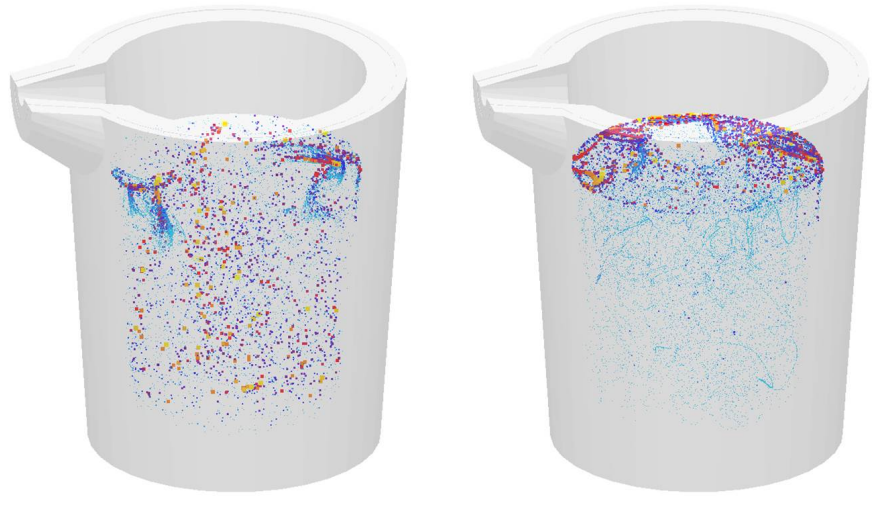

Figure 8: Distribution of inclusions at the end of filling (left) and approx.

10 min after filling (right).

potential). One method to handle this effect is the preconditioning of the melt with a permanent feed of dross particles of a given size during pouring from the ladle. For such an approach the formation mechanisms describing this "dross potential" need to be better understood and quantitatively known. This depends on many parameters, including the chemical composition, $\mathrm{Mg}$ content, treatment process, holding time in the ladle, inoculation etc.

Figure 9 shows the distribution of dross at the end of solidification for two different conditions:

a) Dross formation only due to air entrainment(current model),

b) Additional ladle dross allowed to enter the mold cavity with the melt during pouring.

More inclusions can be seen especially at the top of the bore hole area when ladle dross is allowed to enter the mold cavity.

Another important question to be answered is how much oxygen is entrained during filling and how large the potential is to create oxide inclusions (dross). The amount of oxygen depends on the amount of entrained air and the temperature of the entrained air which doesn't necessarily need to be the pouring temperature of the liquid metal.

\section{Summary}

A new model has been introduced to simulate dross inclusions in ductile iron castings. This model initially only considers the air entrainment due to the free surface turbulence during filling. A first comparison with dross inclusions of a test casting shows a promising agreement with the simulations. 


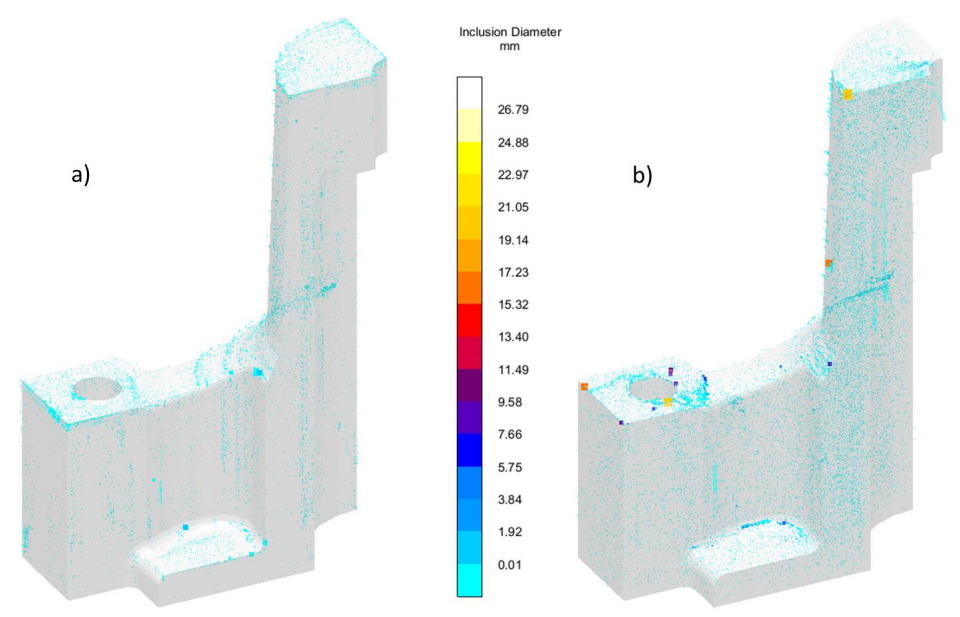

Figure 9: Distribution of dross inclusions at the end of solidification: a) dross formation only due to air entrainment, b) additional ladle dross allowed to enter the mold cavity.

Simulations carried out for large castings showed that the natural convection during solidification seems to play an important role regarding the final distribution of dross inclusions. Furthermore, dross formation cannot completely be avoided even if the filling is quiescent without significant air entrainment. This is because the temperature of the melt usually drops below the equilibrium temperature of the silica reduction by carbon during filling and solidification or is already below that temperature at the beginning of pouring. This implies that based on the metallurgical considerations silica forms and then reacts with the magnesium oxide to form dross inclusions.In addition to oxygen, the initial sulfur content before $\mathrm{Mg}$-treatment influences the amount of dross inclusions [7]. All of these process conditions (Mg-treatment, pouring temperature, contact with sand mold, inoculation etc.) and their influence on dross formation are still not known, at least not quantitatively, and further experimental studies are necessary. The authors are more than willing to discuss any good idea to experimentally quantify dross formation sources.

\section{Courtesy}

Part of this work was done within the R+D project GussTough: "Substitution von Seltenen ErdMetallen zur Entwicklung kaltzäher duktiler Gusseisenwerkstoffe". Supported by the BMBF, Support Code 03X3596E.

\section{References}

[1] Handbuch der Gießerei-Technik, Volume 1, Second Part "Materials".

[2] S.H. Majidi and C. Beckermann: "Modeling of Air Entrainment During Pouring of Metal Castings", Int J Cast Met Res, 30 (2017), 301-315.

[3] J. Ma, A.A. Oberai, D.A. Drew, R.T. Lahey and M. Hyman: "A Comprehensive Sub-Grid Air Entrainment Model for RaNS Modeling of Free-Surface Bubbly Flows", The Journal of Computational Multiphase Flow, 3 (2011), 41-56.

[4] D.A. Ervine, E. McKeough and E.M. Elsawy: "Effect of Turbulence Intensity on the Rate of Air Entrainment by Plunging Water Jets", ICE Proceedings, 1980, Vol. 69, pp. 425-445.

[5] K.J. Sene: “Air Entrainment by Plunging Jets”, Chem Eng Sci, 43 (1988), 2615-2623.

[6] J.W.R. Boyd and J. Varley: "Acoustic Emission Measurement of Low Velocity Plunging Jets to Monitor Bubble Size”, Chem Eng J, 97 (2004), 11-25.

[7] Forschungsbericht E-259, Abschlussbericht zum AiF-Forschungsvorhaben Nr. 11319 N, März 2000: Metallurgische Maßnahmen zur Herstellung einschlußarmer Gußstücke aus Gußeisen mit Kugelgraphit - Vermeidung von Dross. 